\title{
Uso de biossólido em plantios de espécies da Mata Atlântica
}

\author{
Use of biossolid in plantation of atlantic forest species
}

\author{
Marcelo Vinicius da Silva ${ }^{1}$ (D), Guilherme Montandon Chaer $^{2}$ (D), Paulo Sérgio dos Santos Leles ${ }^{1}$ (1), \\ Alexander Silva de Resende ${ }^{2}$ (D), Eduardo Vinicius da Silva ${ }^{1}$ (D), Thaís de Oliveira Campos Barros ${ }^{1}$ (D) \\ ${ }^{1}$ Universidade Federal Rural do Rio de Janeiro - UFRRJ, Seropédica, RJ, Brasil \\ Embrapa Agrobiologia, Seropédica, RJ, Brasil
}

\author{
Como citar: Silva, M. V., Chaer, G. M., Leles, P. S. S., Resende, A. S., Silva, E. V., \& Barros, T. O. C. (2020). Uso de \\ biossólido em plantios de espécies da Mata Atlântica. Scientia Forestalis, 48(126), e2728. \\ https://doi.org/10.18671/scifor.v48n126.16
}

\begin{abstract}
Resumo
O lodo de esgoto tratado, denominado biossólido, contém altos teores de macronutrientes ( $\mathrm{N}, \mathrm{P}, \mathrm{Ca}$ e Mg) e matéria orgânica; por isso, possui grande potencial para fertilização do solo durante a implantação de reflorestamentos. Foram realizados dois experimentos de campo inteiramente casualizados em Planossolo de textura arenosa localizado em Seropédica, RJ. No primeiro, iniciado em abril de 2015, foi avaliado por 19 meses o efeito de quatro doses de biossólido (0, 2, 4 e $8 \mathrm{~L}$ por planta) e uma dose com fertilização mineral (quantidade de nutrientes equivalentes à dose de $4 \mathrm{~L}$ de biossólido) sobre a sobrevivência, o crescimento e a nutrição das espécies Schinus terebinthifolius Radd. (aroeira), Lafoensia glyptocarpa KOEHNE (mirindiba), Inga laurina (Sw.) Willd. (ingá-laurina) e Senna multijuga (Rich.) (pau-cigarra). No segundo experimento, iniciado em março de 2016 e sob os mesmos tratamentos, foram avaliadas por 9 meses as espécies Genipa americana (Vell.) Brenan (jenipapo), Peltophorum dubium (Spreng.) Taub (farinha-seca) e Enterolobium contortisiliquum (Vell.) Morong (orelha-de-negro). Doses crescentes de biossólido aumentaram a taxa de crescimento de $S$. terebinthifolius e $P$. dubium. Entretanto, a maior dose (8 L) reduziu o crescimento de S. multijuga em até $50 \%$, enquanto I. laurina, G. americana e E. contortisiliquum não tiveram seu crescimento afetado pela aplicação de biossólido. A aplicação de biossólido aumentou os teores foliares de S em I. laurina, de P e Ca em L. glyptocarpa e de Mg em S. multijuga, mas reduziu os tores de P em G. americana. Conclui-se que as espécies $S$. terebinthifolius, L. glyptocarpa, P. dubium, I. laurina e S. multijuga se beneficiaram com a dose de biossólido de $4 \mathrm{~L}$ por planta, seja devido à maior taxa de crescimento ou ao maior acúmulo de macronutrientes foliares.
\end{abstract}

Palavras-chave: Lodo de esgoto; Reflorestamento; Restauração florestal; Adubação orgânica.

\begin{abstract}
Treated sewage sludge, called biosolid, contains high levels of macronutrients ( $\mathrm{N}, \mathrm{P}, \mathrm{Ca}$, and $\mathrm{Mg}$ ) and organic matter; therefore, it has great potential for soil fertilization during the implantation of reforestation. Two completely randomized field experiments were carried out in a sandy texture Planosol at Seropédica, RJ. In the first one, initiated in April 2015, the effect of four doses of biosolid (0, 2, 4 and $8 \mathrm{~L}$ per plant) and one dose with mineral fertilization (amount of nutrients equivalent to the dose of $4 \mathrm{~L}$ of biosolid) on the survival, growth and nutrition of the species Schinus terebinthifolius Radd. (aroeira), Lafoensia glyptocarpa KOEHNE (mirindiba), Inga laurina (Sw.) Willd. (ingá-laurina), e Senna multijuga (Rich.) (pau-cigarra). In the second experiment, started in March 2016 and under the same treatments, the following species were evaluated for 9 months: Genipa americana (Vell.) Brenan (jenipapo), Peltophorum dubium (Spreng.) Taub (farinha-seca) and Enterolobium contortisiliquum (Vell.) Morong (orelha-de-negro). Increasing doses of biosolid increased the growth rate of $S$. terebinthifolius and $P$. dubium. However, the
\end{abstract}

Fonte de financiamento: Coordenação de Aperfeiçoamento de Pessoal de Nível Superior - CAPES; Associação Pró-Gestão das Águas da Bacia Hidrográfica do Rio Paraíba do Sul - AGEVAP (Auxílio Financeiro n 020/2016); Companhia Estadual de Águas e Esgotos do Rio de Janeiro - Nova CEDAE.

Conflito de interesse: Nada a declarar.

Autor correspondente: eng.florestal.marcelo@gmail.com

Recebido: 1 dezembro 2017.

Aceito: 29 agosto 2019.

Editor: Francides Gomes Silva Júnior.

(c) (1) Este é um artigo publicado em acesso aberto (Open Access) sob a licença Creative Commons Attribution, que permite uso, distribuição e reprodução cc) em qualquer meio, sem restrições desde que o trabalho original seja corretamente citado. 
higher dose $(8 \mathrm{~L})$ reduced the growth of S. multijuga by up to $50 \%$, while I. laurina, G. americana and E. contortisiliquum did not have their growth affected by the application of biosolids. The application of biosolid increased the leaf content of S in I. laurina, P and Ca in L. glyptocarpa, and $\mathrm{Mg}$ in S. multijuga, but it reduced the content of $\mathrm{P}$ in $\mathrm{G}$. americana. It can be concluded that the species $S$. terebinthifolius, L glyptocarpa, P. dubium, I. laurina and S. multijuga benefited from the dose of biosolid of $4 \mathrm{~L}$ per plant, either due to the higher growth rate or the higher accumulation of macronutrients.

Keywords: Sewage sludge; Reforestation; Forest restoration; Organic fertilization.

\section{INTRODUÇÃO}

No Brasil, como forma de atender a legislação ambiental, os estados e municípios tem acelerado o processo de implantação de Estações de Tratamento de Esgotos (ETE), pois apenas $40,8 \%$ de todo o esgoto produzido é tratado (Brasil, 2016). Por este motivo, é esperado aumento considerável na produção de lodo de esgoto urbano.

O lodo de esgoto é definido pela Norma Técnica Brasileira (NBR) nº 12.209/2011 como suspensão aquosa de componentes minerais e orgânicos separados no sistema de tratamento de esgoto, que para ser descartado necessita de tratamento para redução de sólidos biodegradáveis e odores, estabilização da matéria orgânica, redução de volume e combate a presença de vetores. O lodo de esgoto tratado e estabilizado para o descarte passa a denominar-se biossólido (Abreu, 2014).

Atualmente, a grande parte do biossólido gerado nos grandes centros urbanos possui como destino final os aterros sanitários. O processamento e a disposição final do lodo podem representar até $60 \%$ do custo operacional de uma ETE (Von Sperling, 2001, citado por Godoy, 2013). Diante disso, dentre as alternativas para disposição final do logo de esgoto, aquela com fins agrícola e florestal apresenta-se como uma das mais convenientes (Bettiol \& Camargo, 2006). Como a implantação de povoamentos florestais se dá geralmente em solos com fertilidade reduzida, afetados por anos de agricultura e pecuária, os altos teores de nutrientes e matéria orgânica contidos no biossólido podem ser aproveitados por espécies vegetais sendo, potencialmente, um ótimo insumo para utilização florestal (Andreoli, 1999; Caldeira et al., 2012; Guedes et al., 2006). Para o uso florestal, adiciona-se a vantagem do produto (madeira) não entrar na cadeia alimentar humana, fato que facilita a aceitação do uso desse subproduto por parte de produtores e consumidores (Campos \& Alves, 2008).

Estudos sobre o potencial de utilização do biossólido na agricultura remontam à década de 80 (Bettiol \& Carvalho, 1982). Desde então tem sido estudada sua aplicação com a intenção de obter melhorias no solo (Colodro \& Espíndola, 2006; Colodro et al., 2007; Maio et al., 2011; Maria et al., 2010) e ganhos no desenvolvimento de espécies agronômicas e florestais como a cana-de-açúcar (Silva et al., 2001), o milho (Simonete et al., 2003) e o eucalipto (Rocha et al., 2004). Entretanto, poucos estudos avaliaram o uso de biossólido em plantios de espécies arbóreas nativas utilizadas para restauração florestal. Os trabalhos de Silva \& Pinto (2010) e Lima Filho (2015) são exemplos dos poucos que estudaram o efeito da aplicação do biossólido sobre o crescimento de espécies nativas para restauração florestal. Em ambos os estudos foram demonstrados ganhos em crescimento das espécies estudadas.

Os estudos de Silva \& Pinto (2010) e Lima Filho (2015) abrangeram apenas 12 espécies nativas, o que é um universo reduzido considerando a grande diversidade de espécies arbóreas nativas de vários biomas e que têm sido utilizadas em ações de restauração ambiental no Brasil. Sendo assim, o presente estudo teve como objetivo avaliar o efeito da aplicação de doses de biossólido sobre a sobrevivência, crescimento e nutrição de sete espécies arbóreas da Mata Atlântica em um Planossolo Háplico de textura arenosa.

\section{MATERIAL E MÉTODOS}

O experimento foi conduzido no Campo Experimental da Embrapa Agrobiologia,

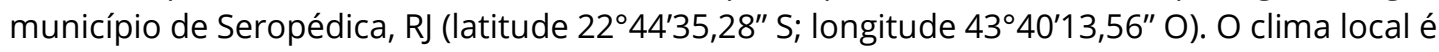
do tipo Aw, segundo classificação de Köppen, com verões chuvosos e invernos secos. No período em que foi conduzido o experimento (abril/2015 a novembro/2016) observou-se 
precipitação acumulada de $1.635 \mathrm{~mm}$ e temperatura média de $23,7^{\circ} \mathrm{C}$, de acordo com as informações da estação meteorológica da Ecologia Agrícola, Seropédica, RJ (Instituto Nacional de Meteorologia, 2017) (Figura 1).

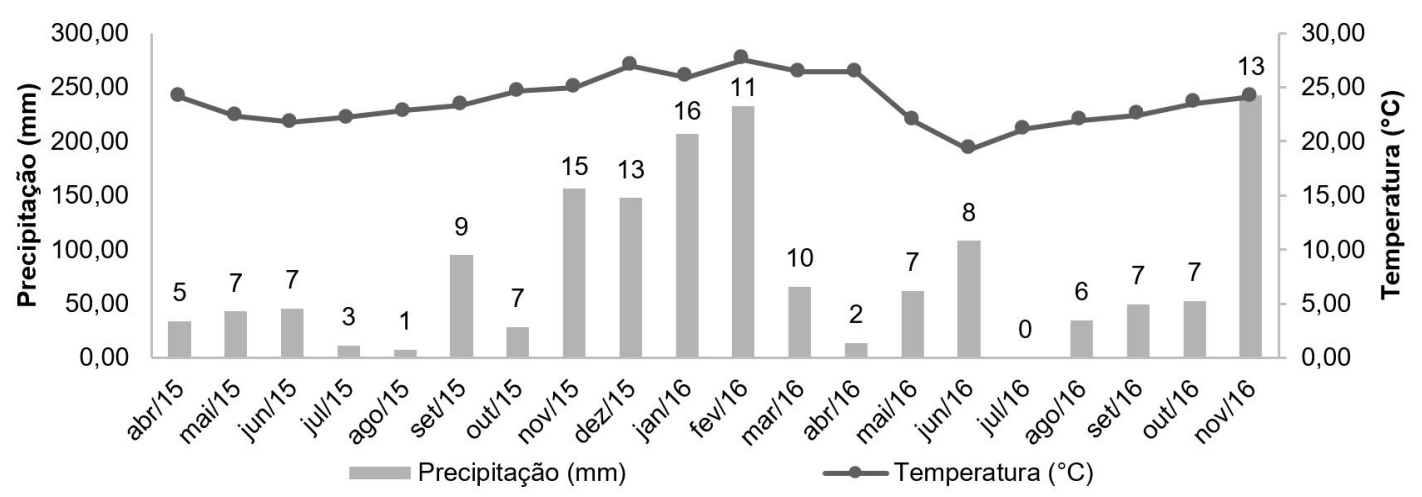

Figura 1: Dados climáticos de abril de 2015 a novembro de 2016 obtidos da estação meteorológica Ecologia Agrícola, Seropédica, RJ (Fonte: Instituto Nacional de Meteorologia, 2017). *Números sobre as barras indicam quantidade de dias com precipitações superiores a $1 \mathrm{~mm}$.

O relevo da área experimental é plano, e o solo foi classificado como Planossolo Háplico, com horizonte superficial bastante arenoso e um horizonte glei em profundidade variada caracterizado pela textura argilosa e pela influência do lençol freático (Santos, 2015).

Foram realizadas coletas de solo nas camadas de 0-10 cm, 10-20 cm e 20-40 cm na área experimental com o objetivo de caracterizá-lo quimicamente. Coletaram-se 10 amostras simples por camada de solo, que posteriormente foram misturadas para formar 3 amostras compostas das 3 camadas, as quais foram analisadas no Laboratório de Química do Solo da Embrapa Agrobiologia em Seropédica, RJ, utilizando metodologias descritas em Empresa Brasileira de Pesquisa Agropecuária (1997) (Tabela 1).

Tabela 1: Características químicas do Planossolo Háplico do local de instalação do experimento no Campo Experimental da Embrapa Agrobiologia, Seropédica, RJ.

\begin{tabular}{|c|c|c|c|c|c|c|c|c|c|}
\hline $\begin{array}{c}\text { Camada } \\
\text { (cm) }\end{array}$ & $\begin{array}{c}\text { pH } \\
\text { (unid.) }\end{array}$ & $\begin{array}{c}P \\
\left(m g \cdot L^{-1}\right)\end{array}$ & $\begin{array}{c}K \\
\left(m g \cdot L^{-1}\right)\end{array}$ & $\begin{array}{c}\mathrm{Ca}^{+2} \\
\left(\mathrm{cmol}_{\mathrm{c} \cdot} \cdot \mathrm{d}^{-3}\right)\end{array}$ & $\begin{array}{c}\mathrm{Mg}^{+2} \\
\left(\mathrm{cmol}_{\mathrm{c} \cdot \mathrm{d}^{-3}}\right)\end{array}$ & $\begin{array}{c}\mathrm{Al}^{-3} \\
\left(\mathrm{cmol}_{\mathrm{c} \cdot \mathrm{d}^{-3}}\right)\end{array}$ & $\begin{array}{c}\mathrm{H}+\mathrm{Al} \\
\left(\mathrm{cmol}_{\left.\mathrm{c} \cdot \mathrm{d}^{-3}\right)}\right.\end{array}$ & $\begin{array}{c}\text { M.O. } \\
(\%)\end{array}$ & $\begin{array}{c}N \\
(\%)\end{array}$ \\
\hline $0-10$ & 5,36 & 8,04 & 31,47 & 0,49 & 0,34 & 0,28 & 4,08 & 0,74 & 0,08 \\
\hline $10-20$ & 5,29 & 6,17 & 21,98 & 0,21 & 0,19 & 0,45 & 3,55 & 0,65 & 0,04 \\
\hline $20-40$ & 5,18 & 4,63 & 11,26 & 0,11 & 0,12 & 0,53 & 2,90 & 0,28 & 0,03 \\
\hline
\end{tabular}

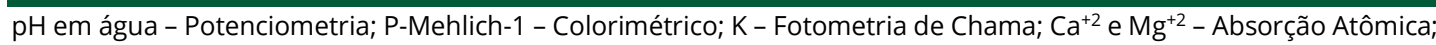
$\mathrm{Al}^{-3}$ - Titulação; H+Al - Titulação; M.O. (\%) - Walkley \& Black; N - Kjeldahl;

Dois experimentos de campo foram instalados para testar doses de biossólido sobre o crescimento de sete espécies arbóreas florestais. Ambos tiveram os mesmos tratamentos, diferenciando-se apenas quanto à data de plantio e as espécies utilizadas. Esse procedimento em duas etapas foi necessário para adequar à disponibilidade de mudas em cada período de plantio.

Na primeira etapa, abril de 2015, mudas de Schinus terebinthifolius Radd. (aroeira), Lafoensia glyptocarpa KOEHNE (mirindiba), Inga laurina (Sw.) Willd. (ingá-laurina) e Senna multijuga (Rich.) (pau-cigarra) foram plantadas no espaçamento $2,5 \times 2,0 \mathrm{~m}$ em covas com dimensões de $20 \times 20 \times 25 \mathrm{~cm}$. O plantio na segunda etapa ocorreu em março de $2016 \mathrm{com}$ o plantio das espécies Genipa americana (Vell.) Brenan (jenipapo), Peltophorum dubium (Spreng.) Taub (farinha seca) e Enterolobium contortisiliquum (Vell.) Morong (orelha-de-negro), também em covas de $20 \times 20 \times 25 \mathrm{~cm}$. As mudas utilizadas foram produzidas em sacolas plásticas de 
aproximadamente $1500 \mathrm{~cm}^{3}$ (9,7 cm diâmetro $\times 20 \mathrm{~cm}$ de altura), exceto mudas de pau-cigarra, que foram produzidas em tubetes de $280 \mathrm{~cm}^{3}$.

O experimento foi montado em esquema inteiramente casualizado com 15 repetições, sendo cada planta considerada uma repetição. Esse delineamento foi escolhido pelo fato da área ser plana e relativamente homogênea, não havendo características de relevo, solo ou padrão de vegetação que justificassem o delineamento em blocos.

As doses de biossólido utilizadas foram de 0, 2, 4 e 8 L.cova $^{-1}$ de plantio. Um controle adicional consistiu na aplicação de fertilização mineral com macronutrientes. As quantidades de N, P e K aplicados no tratamento com fertilização mineral foram calculadas em função dos teores totais de nutrientes presentes na dose de $4 \mathrm{~L}$ do biossólido utilizado, pois foi a dose recomendada por Lima Filho (2015) em estudo com biossólido como adubação orgânica para espécies nativas. Estas quantidades foram equivalentes a $38 \mathrm{~g}$ de $\mathrm{N}, 80 \mathrm{~g}$ de $\mathrm{P}_{2} \mathrm{O}_{5}$ e $7,5 \mathrm{~g}$ de $\mathrm{K}_{2} \mathrm{O}$. Sendo assim, a fertilização do experimento foi composta por três etapas, a primeira no momento do plantio e duas fertilizações de cobertura. No plantio foi aplicado uma mistura formada por $38 \mathrm{~g}$ de sulfato de amônio $(20 \% \mathrm{~N}), 166 \mathrm{~g}$ de fosfato natural $\left(24 \%\right.$ de $\left.\mathrm{P}_{2} \mathrm{O}_{5}\right), 221 \mathrm{~g}$ de superfosfato simples ( $18 \%$ de $\left.\mathrm{P}_{2} \mathrm{O}_{5}\right)$ e $3 \mathrm{~g}$ de cloreto de potássio $\left(58 \%\right.$ de $\left.\mathrm{K}_{2} \mathrm{O}\right)$ por cova de plantio. Adubações complementares de $\mathrm{N}$ e K foram feitas em cobertura aos 3 e aos 6 meses de idade, também apenas no tratamento com fertilização mineral, com a aplicação de $35 \mathrm{~g}$ de ureia e $5 \mathrm{~g}$ de cloreto de potássio por cova em cada aplicação. Essas adubações em cobertura complementaram as doses de $\mathrm{N}$ e K equivalentes à dose de $4 \mathrm{~L}$ de biossólido.

Para o fornecimento de micronutrientes no tratamento com fertilização mineral foram aplicados $20 \mathrm{~g}$ de FTE-BR12 por cova de plantio.

O biossólido utilizado nas duas etapas de plantio foi produzido na Estação de Tratamento de Esgoto da Ilha do Governador (ETIG), Rio de Janeiro. O biossólido foi avaliado quanto aos teores totais de nutrientes no Laboratório de Química do Solo da Embrapa Agrobiologia, por meio da digestão do material em solução nitro-perclórica, semelhantemente ao procedimento de análise de nutrientes em tecido vegetal (Tabela 2).

Tabela 2: Densidade e concentração de macronutrientes, micronutrientes e metais pesados contidos no biossólido da ETIG, RJ.

\begin{tabular}{|c|c|c|c|}
\hline Nutrientes no biossólido ETIG* & Método & Unidade & Valor \\
\hline Densidade & (g) & $\mathrm{kg} \cdot \mathrm{L}^{-1}$ & 0,59 \\
\hline $\mathrm{Ca}(1)$ & (e) & g.kg-1 & 18,11 \\
\hline $\mathrm{Mg}(1)$ & (e) & g. $\mathrm{kg}^{-1}$ & 3,65 \\
\hline $\mathrm{Na}(2)$ & (a) & $\mathrm{mg} \cdot \mathrm{kg}^{-1}$ & 824 \\
\hline$S(2)$ & (a) & g.kg-1 & 9,40 \\
\hline$P(1)$ & (c) & g. $\mathrm{kg}^{-1}$ & 7,37 \\
\hline $\mathrm{K}(1)$ & (d) & g.kg-1 & 1,40 \\
\hline N - Kjeldahl (1) & (b) & $\%$ & 1,62 \\
\hline $\mathrm{N}$ - amoniacal (2) & (b) & $\mathrm{mg} \cdot \mathrm{kg}^{-1}$ & 16,30 \\
\hline $\mathrm{N}$ - Nitrito-Nitrato (2) & (b) & $\mathrm{mg} \cdot \mathrm{kg}^{-1}$ & 629 \\
\hline Sólidos Voláteis (2) & (b) & $\% \mathrm{~m} \cdot \mathrm{m}^{-1}$ & 23,90 \\
\hline Sólidos Totais (2) & (b) & $\% \mathrm{~m} \cdot \mathrm{m}^{-1}$ & 77,60 \\
\hline Umidade, a $60-65^{\circ} \mathrm{C}(2)$ & (b) & $\% \mathrm{~m} \cdot \mathrm{m}^{-1}$ & 22,40 \\
\hline pH (em água 1:10) (2) & (b) & - & 4,30 \\
\hline Carbono Orgânico (2) & (b) & g.kg-1 & 91,70 \\
\hline \multicolumn{4}{|l|}{ Metais Pesados } \\
\hline As (2) & (f) & $\mathrm{mg} \cdot \mathrm{kg}^{-1}$ & $<1 *$ \\
\hline $\mathrm{Cd}(2)$ & $(f)$ & $\mathrm{mg} \cdot \mathrm{kg}^{-1}$ & 1,40 \\
\hline $\mathrm{Pb}(2)$ & $(f)$ & $\mathrm{mg} \cdot \mathrm{kg}^{-1}$ & 58,40 \\
\hline $\operatorname{Cr}(2)$ & $(f)$ & $\mathrm{mg} . \mathrm{kg}^{-1}$ & 21,90 \\
\hline
\end{tabular}


Tabela 2: Continuação...

\begin{tabular}{cccc}
\hline Nutrientes no biossólido ETIG* & Método & Unidade & Valor \\
\hline $\operatorname{Hg}(2)$ & $(\mathrm{f})$ & $\mathrm{mg} \cdot \mathrm{kg}^{-1}$ & $<1 *$ \\
$\mathrm{Ni}(2)$ & $(\mathrm{f})$ & $\mathrm{mg} \cdot \mathrm{kg}^{-1}$ & 12,70 \\
$\mathrm{Se}(2)$ & $(\mathrm{f})$ & $\mathrm{mg} \cdot \mathrm{kg}^{-1}$ & $<1 *$
\end{tabular}

(1) Analisado no Laboratório de Química do Solo da Embrapa Agrobiologia. (2) Fonte: (Campos, 2017); (a) Metais: EPA-SW-846-3051, com determinação por ICP-AES, de acordo com EPA-SW-846-6010. (b) Nitrogênio total: método Kjeldahl; Nitrogênio amoniacal, nitrato e nitrito: destilação por arraste a vapor; Carbono orgânico: digestão com dicromato e determinação volumétrica, Umidade e sólidos voláteis: perda de massa a 60 e $500^{\circ} \mathrm{C}$, respectivamente; pH: determinação em extrato aquoso na proporção 1:10 (resíduo:água) (Andrade \& Abreu, 2006). (c) P: colorimetria. (d) K: fotometria de chama. (e) Ca e Mg: absorção atômica. (f) EPA-SW-846-3051, com determinação por ICP-AES. (g) Instrução Normativa SDA № 17, de 21 de maio de 2007. * Abaixo do limite de quantificação indicado.

O preparo da área experimental consistiu em roçada em área total, abertura das covas ( $50 \mathrm{~cm}$ de diâmetro $\times 50 \mathrm{~cm}$ de profundidade) com broca perfuradora acoplada a tomada de força de um trator e coroamento de $60 \mathrm{~cm}$ de diâmetro. Em seguida, foram aplicadas as doses de biossólido e a de fertilização mineral nas covas correspondente, exceto na testemunha absoluta. Na sequência, o biossólido e o fertilizante mineral foram misturados nas covas utilizando um motocoveador com broca de plantio. As manutenções consistiram de controle de formigas cortadeiras e roçadas periódicas (de 3 a 4 por ano).

Um mês após o plantio foi realizada uma avaliação da mortalidade das espécies e, sendo constatado algum indivíduo morto, o mesmo foi reposto por outro da mesma espécie. Ao final do período de avaliação foi novamente realizada a avaliação de mortalidade das plantas, quantificando-se então o percentual de sobrevivência de cada espécie.

A avaliação de crescimento do primeiro experimento ocorreu aos 4 meses após o plantio e as avaliações seguintes ocorreram em intervalos de 3 meses, até os 19 meses de idade. As avaliações de crescimento das espécies florestais da segunda etapa de plantio ocorreram em intervalos definidos de 3 meses até os 9 meses de idade. As variáveis avaliadas foram altura total, diâmetro ao nível do solo (DNS) (apenas na implantação e na última avaliação) e o comprimento de copa transversal e longitudinal às linhas de plantio, segundo a metodologia descrita por Macho \& Figueiredo Filho (2006) (somente na última avaliação). Através das larguras transversal $\left(\mathrm{L}_{1}\right)$ e longitudinal $\left(\mathrm{L}_{2}\right)$ à linha de plantio, foi calculado a área de copa $\left(\mathrm{C}_{\mathrm{i}}\right)$ de cada indivíduo, sempre considerando a copa das espécies florestais como uma elipse perfeita, através da equação:

$C_{i}=\pi \times\left[\left(\frac{L_{1}+L_{2}}{4}\right)^{2}\right]$

Em 24 de novembro de 2016 foi realizada uma coleta de folhas das espécies em cada tratamento para obtenção do teor de macronutrientes. Para cada tratamento, foram amostrados 4 indivíduos que mais se aproximavam da média de DNS do tratamento. Foram coletadas folhas do terço médio da copa dos indivíduos. Posteriormente, as amostram foram enviadas para o Instituto Agronômico de Campinas (IAC) para serem analisadas, de acordo com a metodologia descrita por Bataglia et al. (1983).

Foram elaboradas curvas de crescimento em altura ao longo das medições tendo como referência a altura de cada muda no momento do plantio. Desse modo, as curvas representaram o incremento cumulativo da altura ao longo do período de avaliação.

Após verificadas variação linear, homogeneidade de variância e normalidade dos erros por meio de análise gráfica da distribuição de resíduos e de QQ-plots, os dados foram submetidos à análises de regressão linear para avaliar a relação entre as variáveis de crescimento, teores de nutrientes foliares e as doses de biossólido. Entretanto, não foi possível ajustar modelos significativos para a maioria das variáveis obtidas dentre as espécies testadas. Os poucos modelos significativos apresentaram baixos coeficientes de determinação. Logo, nos casos em que não foi possível obter ajustes de curvas significativos, 
foi realizada a análise de variância seguida da aplicação do teste de Dunnett para comparar o efeito do tratamento controle em relação aos tratamentos com aplicação de diferentes doses de biossólido e o tratamento com fertilizante mineral. Esse teste consiste na comparação de cada tratamento experimental em relação a um único tratamento controle por meio do cálculo da estatística t de Student. As análises foram realizadas no software S-PLUS 8.0 (Insightful Corp.).

\section{RESULTADOS E DISCUSSÃO}

\section{Taxa de sobrevivência das mudas}

A aplicação de biossólido reduziu a taxa de sobrevivência das plantas de l. laurina e S. multijuga (Tabela 3). A maior dose de biossólido também reduziu a taxa de sobrevivência de L. glyoptocarpa, mas as doses inferiores ( $2 \mathrm{~L} \mathrm{e} 4 \mathrm{~L}$ ) aumentaram a sobrevivência dessa espécie. Para as demais espécies, não há evidência de que o biossólido tenha promovido aumento ou redução da taxa de sobrevivência.

A fertilização mineral também afetou negativamente a sobrevivência de I. laurina, L. glyoptocarpa e S. multijuga, embora de forma mais acentuada (Tabela 3). A taxa de sobrevivência dessas espécies foi de $20 \%$, $33 \%$ e $0 \%$, respectivamente.

Tabela 3: Percentual de sobrevivência de sete espécies florestais da Mata Atlântica aos 9 meses (P. dubium, G. americana e E. contortisiliquum) e aos 19 meses (S. terebinthifolius, I. laurina, L. glyptocarpa e S. multijuga) após o plantio, em função da aplicação de doses de biossólido ou fertilização mineral (FM).

\begin{tabular}{|c|c|c|c|c|c|}
\hline \multirow{3}{*}{ Espécie } & \multicolumn{4}{|c|}{ Dose de biossólido } & \multirow{2}{*}{ FM } \\
\hline & $\mathbf{O L}$ & $2 \mathrm{~L}$ & $4 \mathrm{~L}$ & $8 \mathrm{~L}$ & \\
\hline & \multicolumn{5}{|c|}{ 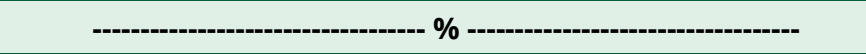 } \\
\hline \multicolumn{6}{|c|}{ Espécies com 19 meses de idade } \\
\hline S. terebinthifolius & 93 & 93 & 100 & 100 & 87 \\
\hline I. laurina & 73 & 67 & 53 & 40 & 20 \\
\hline L. glyptocarpa & 73 & 93 & 87 & 60 & 33 \\
\hline S. multijuga & 60 & 47 & 47 & 40 & 0 \\
\hline Média & 75 & 75 & 72 & 60 & 35 \\
\hline \multicolumn{6}{|c|}{ Espécies com 9 meses de idade } \\
\hline P. dubium & 100 & 100 & 100 & 100 & 87 \\
\hline G. americana & 93 & 100 & 93 & 87 & 80 \\
\hline E. contortisiliquum & 93 & 93 & 93 & 93 & 93 \\
\hline Média & 95 & 98 & 95 & 93 & 87 \\
\hline
\end{tabular}

O baixo percentual de sobrevivência dessas espécies, tanto no tratamento com fertilização mineral quanto no da maior dose de biossólido, pode estar associado à baixa precipitação apresentada no mês que sucedeu o plantio (Figura 1). Um possível aumento de solutos no solo devido à aplicação dos fertilizantes minerais e do biossólido na maior dose pode ter reduzido o potencial osmótico do solo ao ponto de limitar a capacidade de absorção de água pelas raízes das plantas durante o período de déficit hídrico após o plantio. Salienta-se que após o plantio não foi feita irrigação das plantas.

Sabe-se que a salinidade no solo, além de poder afetar as plantas em funções osmóticas, pode provocar toxidez, afetar a cadeia respiratória, a fotofosforilação, prejudicar a assimilação de nitrogênio e o metabolismo de proteínas (Munns, 2002; Tester \& Davenport, 2003). A hipótese de mortalidade de plantas devido à redução do potencial osmótico do solo é plausível pelo fato de que no plantio da segunda etapa, apesar de se tratar de espécies 
distintas, houve boa disponibilidade hídrica que resultou em elevada taxa de sobrevivência das plantas em todos os tratamentos.

A menor taxa de sobrevivência foi observada na espécie S. multijuga (Tabela 3), o que provavelmente esteja relacionado ao tipo de recipiente utilizado na produção das mudas, ou seja, em tubetes de $280 \mathrm{~cm}^{3}$. O menor volume de substrato pode tornar a planta mais vulnerável após o plantio sob ocorrência de déficit hídrico. Por outro lado, a alta sobrevivência de S. terebinthifolius no plantio da primeira etapa demonstra uma melhor adaptação dessa espécie às condições de déficit hídrico e salinidade do solo decorrente da aplicação da fertilização mineral. S. terebinthifolius é reconhecidamente uma espécie com capacidade de sobreviver em ambientes adversos. Embora não seja seu ambiente natural, essa espécie ocorre na Caatinga (Baggio, 1988), bioma com uma estação seca prolongada, chuvas irregulares e altas temperaturas. S. terebinthifolius é capaz ainda de invadir áreas de mangue, o que demonstra sua alta adaptabilidade a ambientes salinos (Dawkins \& Esiobu, 2016).

\section{Crescimento em diâmetro e altura da mudas}

O aumento da dose de biossólido favoreceu o incremento em altura de S. terebinthifolius quando comparado ao tratamento controle, efeito que se intensificou a partir dos 13 meses após o plantio (Figura 2). Espécies que não apresentaram resposta positiva de crescimento em altura em função da aplicação do biossólido foram L. glyptocarpa e S. multijuga. A maior dose de biossólido ( 8 L) favoreceu a espécies I. laurina, porém, reduziu sensivelmente o incremento em altura de S. multijuga (Figura 2).

A aplicação de fertilização mineral não afetou significativamente o crescimento em altura de I. laurina e L. glyptocarpa, mas reduziu o de S. terbinthifolius e de S. multijuga (Figura 2).
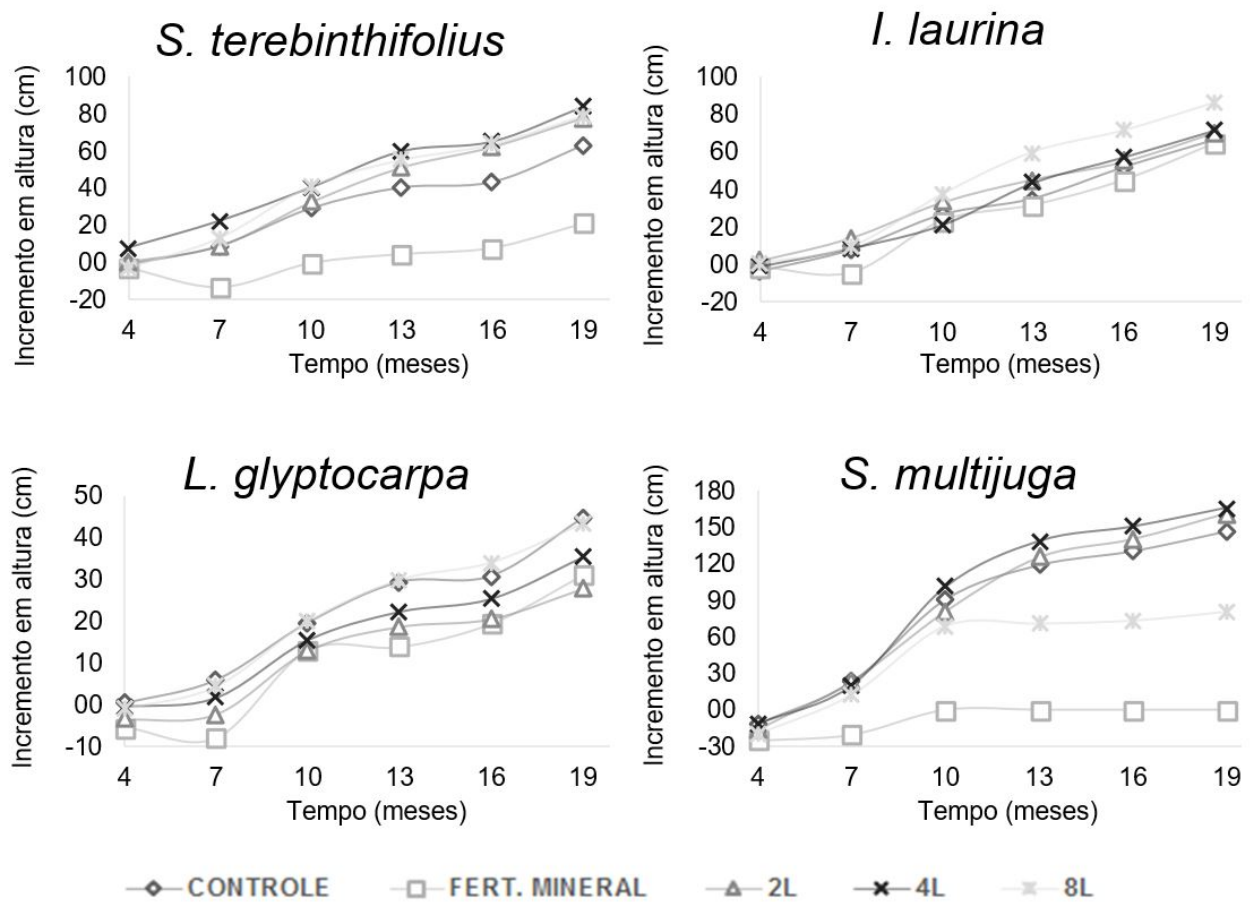

Figura 2: Incremento cumulativo em altura de quatro espécies ao longo de 19 meses de período de crescimento, sob diferentes doses de biossólido $(0,2,4$ e $8 \mathrm{~L})$ e controle com fertilização mineral.

No segundo experimento, as doses de 4 e 8 L/cova de biossólido beneficiaram o incremento em altura de P. dubium (Figura 3, Tabela 4). A espécie G. americana apresentou incremento em altura negativo para o tratamento com fertilizante mineral, enquanto que E. Contortisiliquum, para o mesmo tratamento, foi beneficiado e apresentou incremento em altura superior aos tratamentos controle ou com biossólido (Figura 3). 

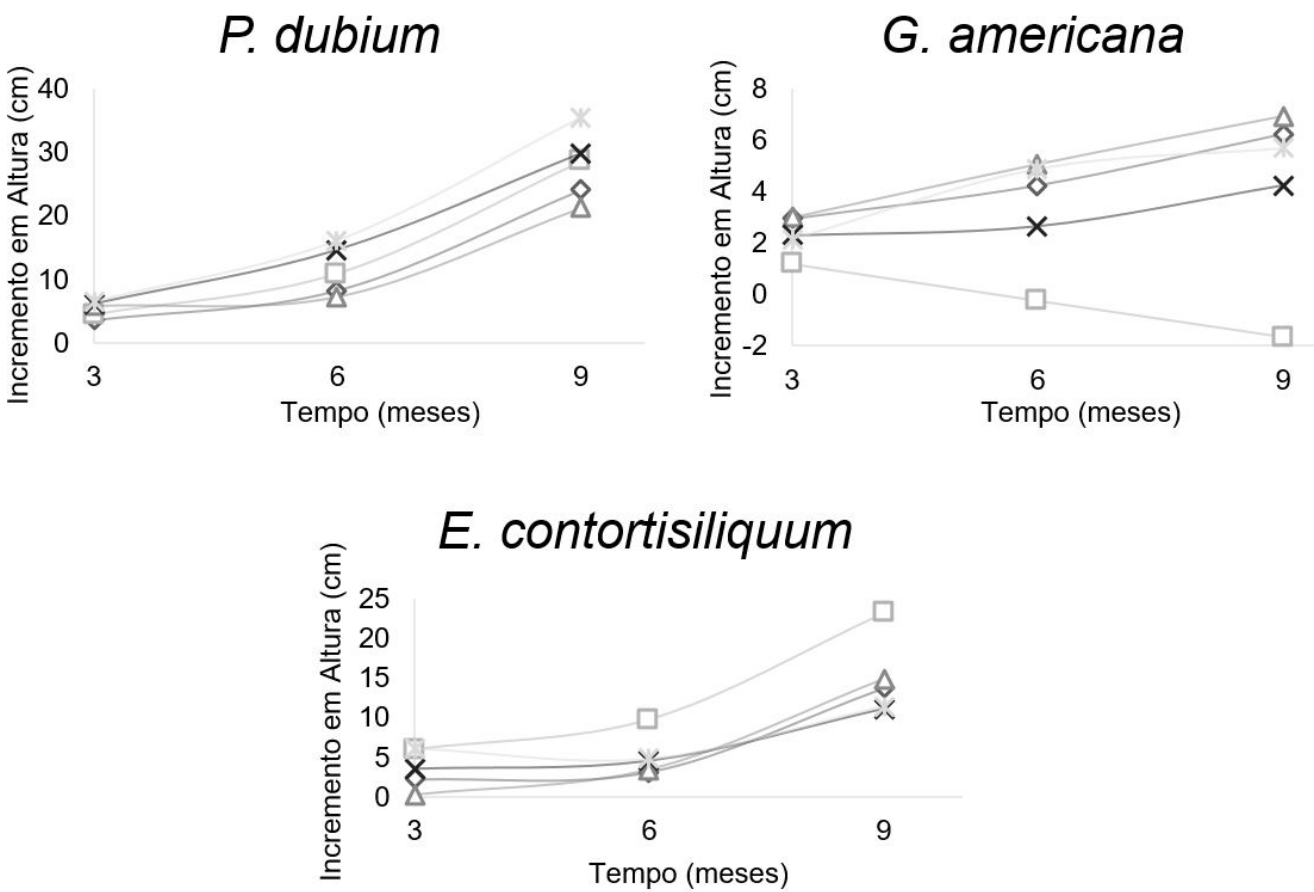

$\because$ CONTROLE

FERT. MINERAL

$\triangle-2 \mathrm{~L}$

$x-4 \mathrm{~L}$

$8 \mathrm{~L}$

Figura 3: Incremento cumulativo em altura de 3 espécies ao longo de 9 meses de período de crescimento, sob diferentes doses de biossólido $(0,2,4$ e $8 \mathrm{~L}$ ) e controle com fertilização mineral.

Tabela 4: Taxas de incremento médio em altura e diâmetro ao nível do solo (DNS) e área de copa de espécies florestais do bioma Mata Atlântica adubadas com 0, 2, 4, ou $8 \mathrm{~L}$ de biossólido ou com fertilizantes minerais (FM). Os asteriscos seguidos das médias indicam diferença significativa $(p<0,05)$ da comparação par a par entre o tratamento controle $(0 \mathrm{~L})$ e cada nível de adubação com biossólido ou fertilizante mineral.

\begin{tabular}{|c|c|c|c|c|}
\hline Espécie (idade) & Trat. & $\begin{array}{c}\text { Incremento } \\
\text { altura } \\
\text { (cm/mês) }\end{array}$ & $\begin{array}{l}\text { Incremento } \\
\text { DNS } \\
\text { (mm/mês) }\end{array}$ & $\begin{array}{c}\text { Área de copa } \\
\qquad\left(\mathrm{m}^{2}\right)\end{array}$ \\
\hline \multirow{5}{*}{$\begin{array}{l}\text { S. terebinthifolius } \\
\text { (19 meses) }\end{array}$} & $O \mathrm{~L}$ & 3,62 & 1,09 & 2,76 \\
\hline & $2 \mathrm{~L}$ & 4,14 & 1,26 & $4,01^{(0,07)}$ \\
\hline & $4 \mathrm{~L}$ & $4,42^{(0,19)}$ & 1,38 * & $3,65^{(0,18)}$ \\
\hline & $8 \mathrm{~L}$ & 4,17 & $1,44 *$ & 3,59 \\
\hline & FM & $2,18^{*}$ & $0,84^{*}$ & $1,84^{*}$ \\
\hline \multirow{5}{*}{$\begin{array}{l}\text { I. laurina } \\
\text { (19 meses) }\end{array}$} & $0 \mathrm{~L}$ & 3,45 & 1,04 & 1,31 \\
\hline & $2 \mathrm{~L}$ & 4,19 & 1,10 & 1,11 \\
\hline & $4 \mathrm{~L}$ & 3,53 & 1,17 & 1,24 \\
\hline & $8 \mathrm{~L}$ & 4,17 & 1,17 & 1,58 \\
\hline & FM & 3,09 & 0,98 & 0,82 \\
\hline \multirow{5}{*}{$\begin{array}{l}\text { L. glyptocarpa } \\
\text { (19 meses) }\end{array}$} & $0 \mathrm{~L}$ & 2,24 & 0,84 & 0,60 \\
\hline & $2 \mathrm{~L}$ & $1,55^{(0,15)}$ & 0,73 & 0,43 \\
\hline & $4 \mathrm{~L}$ & 2,06 & 0,73 & 0,51 \\
\hline & $8 \mathrm{~L}$ & 2,81 & 0,89 & 0,76 \\
\hline & FM & 1,61 & $0,60^{(0,15)}$ & 0,38 \\
\hline \multirow{5}{*}{$\begin{array}{l}\text { S. multijuga } \\
\text { (19 meses) }\end{array}$} & $0 \mathrm{~L}$ & 7,71 & 1,59 & 3,12 \\
\hline & $2 \mathrm{~L}$ & 8,35 & 1,64 & 2,74 \\
\hline & $4 \mathrm{~L}$ & 8,71 & 1,88 & 3,74 \\
\hline & $8 \mathrm{~L}$ & $4,05 *$ & $0,93^{(0,12)}$ & $1,18^{*}$ \\
\hline & FM & - & - & - \\
\hline
\end{tabular}


Tabela 4: Continuação...

\begin{tabular}{|c|c|c|c|c|}
\hline Espécie (idade) & Trat. & $\begin{array}{l}\text { Incremento } \\
\text { altura } \\
\text { (cm/mês) }\end{array}$ & $\begin{array}{c}\text { Incremento } \\
\text { DNS } \\
\text { (mm/mês) }\end{array}$ & $\begin{array}{c}\text { Área de copa } \\
\left(\mathrm{m}^{2}\right)\end{array}$ \\
\hline \multirow{5}{*}{$\begin{array}{l}\text { P. dubium } \\
\text { (9 meses) }\end{array}$} & $0 \mathrm{~L}$ & 2,34 & 1,01 & 0,43 \\
\hline & $2 \mathrm{~L}$ & 2,85 & 0,96 & 0,46 \\
\hline & $4 \mathrm{~L}$ & $3,33^{*}$ & 1,10 & 0,53 \\
\hline & $8 \mathrm{~L}$ & $4,71 *$ & $1,28^{(0,12)}$ & $0,56^{(0,11)}$ \\
\hline & FM & $2,98^{(0,17)}$ & 0,86 & 0,54 \\
\hline \multirow{5}{*}{$\begin{array}{l}\text { G. americana } \\
\text { (9 meses) }\end{array}$} & $0 \mathrm{~L}$ & 0,73 & 0,12 & 0,08 \\
\hline & $2 \mathrm{~L}$ & 0,77 & 0,12 & 0,08 \\
\hline & $4 \mathrm{~L}$ & 0,81 & 0,13 & 0,06 \\
\hline & $8 \mathrm{~L}$ & 0,70 & 0,14 & 0,07 \\
\hline & FM & 0,74 & 0,07 & $0,05^{(0,17)}$ \\
\hline \multirow{5}{*}{$\begin{array}{l}\text { E. contortisiliquum } \\
\text { (9 meses) }\end{array}$} & $\mathrm{OL}$ & 1,64 & 0,48 & 0,16 \\
\hline & $2 \mathrm{~L}$ & 1,95 & 0,44 & 0,17 \\
\hline & $4 \mathrm{~L}$ & 1,79 & 0,35 & 0,14 \\
\hline & $8 \mathrm{~L}$ & 1,54 & 0,41 & 0,16 \\
\hline & FM & $2,85^{*}$ & 0,46 & 0,19 \\
\hline
\end{tabular}

Expoentes numéricos entre parênteses indicam $0,05<p<0,20$.

Plantas de S. terebinthifolius, I. laurina e L. glyptocarpa apresentaram incrementos negativos na altura entre o quarto e sétimo mês de crescimento no tratamento com fertilização mineral (Figura 2). Esse fato ocorreu devido à morte e quebra dos ponteiros causado pelo período de estiagem descrito anteriormente. No caso de G. Americana, esse efeito parece ter sido mais intenso e perdurou durante todo o período de avaliação (Figura 3).

O efeito positivo do aumento das doses de biossólido sobre variáveis de crescimento pôde ser observado em S. terebinthifolius (incremento em DNS) e $P$. dubium (incremento em altura e DNS e área de copa) (Tabela 4). A dose de $8 \mathrm{~L}$ de biossólido resultou no maior incremento em DNS em $S$. terebinthifolius e nos maiores incrementos de todas as variáveis de crescimento de $P$. dubium. As espécies I. laurina, L. glyptocarpa, G. americana e E. contortisiliquum não apresentaram resposta à aplicação de biossólido. S. multijuga também não respondeu ao biossólido até a dose de $4 \mathrm{~L}$, mas a aplicação da dose de $8 \mathrm{~L}$ reduziu em aproximadamente $50 \%$ o crescimento de S. multijuga (Tabela 4).

A aplicação da fertilização mineral reduziu significativamente todas variáveis de crescimento de S. terebinthifolius $(p<0,05)$. Tendência similar foi observada para $G$. americana (área de copa), L. glyptocarpa (incremento em DNS) e $P$. dubium (incremento em altura) $(p<0,20)$. Ao contrário, E. contortisiliquum mostrou resposta positiva à fertilização mineral, expressa especialmente na variável incremento em altura que foi cerca de $70 \%$ superior ao controle (Tabela 4).

A aplicação de biossólido no solo, além de fornecer nutrientes às plantas, atua na melhoria da estruturação das partículas do solo, favorecendo a infiltração de água e o desenvolvimento do sistema radicular (Abreu, 2014; Andreoli et al., 2001; Barbosa \& Tavares Filho, 2006). Neste trabalho, as espécies S. terebinthifolius, L. glyptocarpa, P. dubium responderam de forma positiva à aplicação de biossólido na cova de plantio na maioria dos parâmetros silviculturais mensurados. Lima Filho (2015), em Latossolo Vermelho Amarelo da região serrana do Rio de Janeiro, constatou que a aplicação de $3 \mathrm{~L}$ de biossólido na cova de plantio resultou no maior crescimento das espécies P. dubium, Ceiba speciosa e Sapindus saponaria em comparação a plantas não adubadas ou adubadas com fertilizante mineral.

As espécies I. laurina e G. americana não apresentaram resposta à aplicação de biossólido em nenhuma das variáveis de crescimento avaliadas (Tabela 4). Em estudo anterior conduzido por Silva \& Pinto (2010) utilizando as espécies Luehea divaricata, Cedrela fissilis, Lonchocarpus muehlbergianus, Erythrina falcate, Aspidosperma polyneuron, Erythroxylum deciduum e Araucaria angustifolia, também não foi encontrada diferenças significativas de crescimento entre 
tratamentos com aplicação de biossólido ou fertilização mineral e a testemunha absoluta, 36 meses após o plantio.

Esse conjunto de resultados demonstra que há uma grande variabilidade de resposta à fertilização entre espécies arbóreas nativas. Como visto, várias delas são pouco responsivas à fertilização, provavelmente por apresentarem alto grau de rusticidade e adaptação a solos de baixa fertilidade.

Silva et al. (1997) sugeriram que a resposta ao potássio de várias espécies florestais está associada ao grupo sucessional a que pertence. Segundo esses autores, as espécies mais responsivas ao potássio foram aquelas com maiores taxa de crescimento e classificadas como pioneiras. Segundo Gonçalves et al. (2000), espécies nativas dos estágios iniciais de sucessão apresentam taxas de crescimento superiores às de final de sucessão. Logo, quanto maiores as taxas de crescimento, maiores são as demandas por nutrientes, especialmente na fase inicial de crescimento (Paiva, 2005).

Os resultados do presente estudo não são conclusivos quanto a resposta de grupos sucessionais à fertilização. Enquanto a pioneira S. terebinthifolius apresentou resposta à aplicação de biossólido, o mesmo não ocorreu para a pioneira S. Multijuga, ambas de crescimento rápido. Já a espécie I. laurina, secundária inicial, não apresentou resposta significativa ao tratamento com biossólido (Figura 2).

\section{Estado nutricional das plantas}

Não foi possível realizar análise de comparação do teor nutricional entre as dosagens de biossólido com o tratamento com fertilizantes minerais para S. multijuga, pois o tratamento com fertilizante mineral apresentou $100 \%$ de mortalidade.

Os maiores teores foliares de $\mathrm{S}$ em I. laurina estiveram associados à aplicação da dose de $2 \mathrm{~L}$ de biossólido por cova (Tabela 5). Para L. glyptocarpa, o maior teor foliar de $\mathrm{P}$ foi associado à dose de $4 \mathrm{~L}$ e ao tratamento com fertilização mineral; para o $\mathrm{Ca}$, o maior teor foliar ocorreu na dose de 8 L. S. multijuga apresentou o maio teor foliar de Mg na dose de 4 L. Ao contrário, os teores de P foliar em G. americana decresceram com o aumento da dose de biossólido (Tabela 5).

Tabela 5: Teor de macronutrientes foliares aos 19 meses para as espécies S. terebinthifolius, I. laurina, L. glyptocarpa e S. multijuga. Os asteriscos seguidos das médias indicam diferença significativa $(p<0,05)$ da comparação par a par entre o tratamento controle $(0 \mathrm{~L})$ e cada nível de adubação com biossólido ou fertilizante mineral.

\begin{tabular}{|c|c|c|c|c|c|c|c|}
\hline \multirow{2}{*}{ Espécie } & \multirow{2}{*}{ Trat. } & $\mathbf{N}$ & $\mathbf{P}$ & $\mathbf{K}$ & $\mathrm{Ca}$ & Mg & $\mathbf{S}$ \\
\hline & & \multicolumn{6}{|c|}{ - } \\
\hline \multirow{5}{*}{ S. terebinthifolius } & $0 \mathrm{~L}$ & 15,1 & 1,72 & 9,14 & 6,21 & 1,84 & 2,92 \\
\hline & $2 \mathrm{~L}$ & 14,2 & 2,16 & 9,94 & 6,59 & 2,02 & 2,97 \\
\hline & $4 \mathrm{~L}$ & 15,5 & 1,94 & 9,14 & 7,44 & 2,04 & 3,00 \\
\hline & $8 \mathrm{~L}$ & 15,0 & 1,66 & 8,25 & $7,95^{(0,14)}$ & 2,08 & 2,80 \\
\hline & FM & 15,9 & $2,23^{(0,20)}$ & 10,85 & 6,83 & $2,33^{(0,16)}$ & 3,24 \\
\hline \multirow{5}{*}{ I. laurina } & $0 \mathrm{~L}$ & 20,0 & 1,35 & 5,28 & 3,63 & 1,08 & 1,06 \\
\hline & $2 \mathrm{~L}$ & $24,0^{(0,13)}$ & 1,41 & 5,34 & $4,60^{(0,11)}$ & 1,02 & $1,26 *$ \\
\hline & $4 \mathrm{~L}$ & 22,0 & 1,37 & $4,45^{(0,12)}$ & 4,17 & $0,88^{(0,07)}$ & $1,20 *$ \\
\hline & $8 \mathrm{~L}$ & 21,8 & 1,39 & $4,57^{(0,18)}$ & 3,43 & 0,97 & 1,14 \\
\hline & FM & 21,1 & 1,29 & 5,02 & 4,17 & 0,99 & 1,05 \\
\hline \multirow{5}{*}{ L. glyptocarpa } & $O L$ & 11,54 & 1,64 & 9,38 & 5,47 & 2,21 & 2,24 \\
\hline & $2 \mathrm{~L}$ & 12,27 & $2,38^{(0,11)}$ & 9,57 & $8,26 * * *$ & 2,05 & 2,22 \\
\hline & $4 \mathrm{~L}$ & $13,09^{(0,13)}$ & $2,43^{(0,09)}$ & 9,95 & $8,36 * * *$ & 2,11 & 2,38 \\
\hline & $8 \mathrm{~L}$ & $13,07^{(0,13)}$ & 2,01 & 7,33 & $9,40 * * *$ & 2,22 & 1,82 \\
\hline & FM & 11,97 & $2,46^{(0,08)}$ & 10,77 & $7,41 *$ & 1,89 & 2,01 \\
\hline
\end{tabular}


Tabela 5: Continuação...

\begin{tabular}{|c|c|c|c|c|c|c|c|}
\hline \multirow{2}{*}{ Espécie } & \multirow{2}{*}{ Trat. } & $\mathbf{N}$ & $\mathbf{P}$ & $\mathbf{K}$ & $\mathrm{Ca}$ & Mg & $\mathbf{S}$ \\
\hline & & \multicolumn{6}{|c|}{ 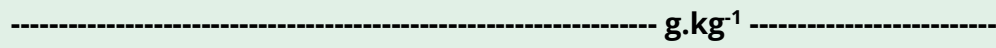 } \\
\hline \multirow{5}{*}{ S. multijuga } & $0 \mathrm{~L}$ & 19,40 & 1,80 & 6,91 & 6,80 & 1,79 & 1,58 \\
\hline & $2 \mathrm{~L}$ & 18,83 & $2,58^{(0,15)}$ & 7,11 & $9,97^{(0,20)}$ & 2,15 & 1,70 \\
\hline & $4 \mathrm{~L}$ & 20,41 & 2,07 & 6,99 & $10,47^{(0,14)}$ & $2,56 *$ & 1,48 \\
\hline & $8 \mathrm{~L}$ & 18,14 & 2,37 & 6,89 & $11,01^{(0,10)}$ & 1,91 & 1,84 \\
\hline & $\mathrm{FM}$ & - & - & - & - & - & - \\
\hline
\end{tabular}

Expoentes numéricos entre parênteses indicam 0,05 $<p<0,20$.

Apesar das informações sobre exigência nutricional de espécie nativas serem escassas (Carpanezzi et al., 1976), Gonçalves (1994) afirma que espécies da Mata Atlântica dependem muito da fertilidade do solo. No caso desse estudo, o solo pobre (Tabela 1) permitiu evidenciar, com adição das doses de biossólido, bem como do fertilizante mineral, alteração nos teores foliares de alguns macronutrientes de determinadas espécies.

A aplicação de fertilizantes minerais, em conteúdo equivalente ao presente na dose de $4 \mathrm{~L}$ de biossólido, aumentou significativamente o teor de $\mathrm{P}$ foliar de L. glyptocarpa em relação ao controle (dose $0 \mathrm{~L}$ de biossólido), mas não diferiu dos tratamentos com aplicação de 2,4 ou $8 \mathrm{~L}$ de biossólido. Os teores de $\mathrm{P}$ foliar também foram maiores em plantas de G. americana e E. contortisiliquum que receberam fertilização mineral em relação a todos os demais tratamentos. Para P. dubium, o teor de P foliar no tratamento com fertilizante mineral foi superior apenas aos com aplicação de 0,2 ou $4 \mathrm{~L}$ de biossólido, não diferindo do tratamento com aplicação de $8 \mathrm{~L}$ de biossólido por cova de plantio (Tabela 6). A fertilização mineral aumentou os teores de N foliar apenas de G. americana e de P. Dubium (Tabela 6).

Tabela 6: Teor de macronutrientes foliares aos 9 meses para as espécies $P$. dubium, G. americana e E. contortisiliquum. Os asteriscos seguidos das médias indicam o nível de significância da comparação entre o tratamento controle $(0 \mathrm{~L})$ e cada nível de adubação com biossólido ou fertilizante mineral.

\begin{tabular}{|c|c|c|c|c|c|c|c|}
\hline \multirow{2}{*}{ Espécie } & \multirow{2}{*}{ Tratamento } & $\mathbf{N}$ & $\mathbf{P}$ & $\mathbf{K}$ & $\mathrm{Ca}$ & Mg & $\mathbf{S}$ \\
\hline & & \multicolumn{6}{|c|}{ 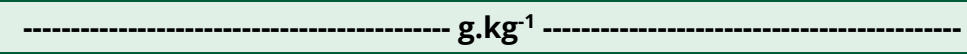 } \\
\hline \multirow{5}{*}{ P. dubium } & $0 \mathrm{~L}$ & 16,88 & 1,15 & 6,80 & 4,485 & 1,66 & 2,62 \\
\hline & $2 \mathrm{~L}$ & 15,97 & 1,12 & 5,68 & $6,29^{(0,09)}$ & 2,02 & 2,97 \\
\hline & $4 \mathrm{~L}$ & 16,38 & 1,17 & 6,78 & 5,70 & 1,84 & 3,16 \\
\hline & $8 \mathrm{~L}$ & 16,58 & $1,41^{(0,16)}$ & 6,47 & 5,38 & 1,86 & $3,37^{(0,16)}$ \\
\hline & FM & $28,64 *$ & $1,50^{(0,08)}$ & 6,68 & 4,01 & 1,49 & 2,97 \\
\hline \multirow{5}{*}{ G. americana } & $0 \mathrm{~L}$ & 16,27 & 2,39 & 11,25 & 4,83 & 3,07 & 1,63 \\
\hline & $2 \mathrm{~L}$ & 19,82 & 2,10 & 9,79 & 3,74 & 3,14 & 1,79 \\
\hline & $4 \mathrm{~L}$ & 16,66 & 1,86 & 10,75 & 4,89 & 2,73 & 1,92 \\
\hline & $8 \mathrm{~L}$ & 14,75 & $1,26^{(0,09)}$ & $6,98^{(0,14)}$ & 4,70 & $4,23^{(0,11)}$ & 1,84 \\
\hline & FM & $22,55^{(0,08)}$ & 3,24 & 11,87 & 5,66 & 2,99 & 2,08 \\
\hline
\end{tabular}

$\mathrm{p}<0,05$. Expoentes numéricos entre parênteses indicam $0,05<p<0,20$.

Guedes \& Poggiani (2003) não verificaram aumento significativo no teor foliar de P até os 6 meses de idade num plantio de Eucalyptus grandis, provavelmente devido a aplicação do biosólido ter sido realizada nas entrelinhas de plantio, uma vez que o P está imobilizado na matéria orgânica e possui baixa mobilidade no solo, e portanto, não se encontra prontamente disponível para absorção pelo sistema radicular das plantas. Apesar da fertilização de plantio com biossólido nesse trabalho ter ocorrido de forma localizada na cova, a mineralização do $P$ 
parece não ter ocorrido de forma satisfatória para elevar os teores desse nutriente nas folhas das espécies florestais nativas, com exceção na espécie $P$. dubium. Embora os teores nutricionais foliares terem sido menores nos tratamentos com biossólido, essa diferença não se traduziu em menor crescimento, principalmente para as espécies $S$. terebinthifolius, L. glyptocarpa e $P$. dubium, sugerindo um aumento da eficiência de uso de nutrientes por essas espécies.

Em geral, não houveram alterações nos teores foliares de $\mathrm{K}, \mathrm{Ca}, \mathrm{Mg}$ e $\mathrm{S}$ das espécies avaliadas devido a aplicação de fertilização mineral em relação ao tratamento controle, à exceção do aumento do teor de Ca foliar de L. glyptocarpa.

Normalmente, os valores de K são baixos no biossólido devido ao processo de lavagem no tratamento do material (Berton \& Nogueira, 2010). Silva et al. (2008a), conduziram experimento com fertilização para Eucalyptus grandis em Itatinga, SP, em um Latossolo-Amarelo de baixa fertilidade, no qual encontrou resultados insuficientes de $K$ nos tratamentos com biossólido; portanto, os autores recomendam que esse tipo de fertilização seja complementado com fontes de K e B antes da aplicação.

Guedes \& Poggiani (2003), avaliando o efeito da aplicação de biossólido em um povoamento jovem de Eucalyptus grandis, constataram que plantas que receberam biossólido em cobertura após o plantio apresentaram teores foliares mais elevados de N, P, Ca, S e mais baixos de $\mathrm{Mg}$ e $\mathrm{Mn}$ em relação a plantas que receberam fertilização mineral. Em outro estudo com a mesma espécie, Silva et al. (2008b) mostraram que a aplicação de $30 \mathrm{t} \mathrm{ha} \mathrm{a}^{-1}$ promoveu o aumento dos teores foliares de P, Ca e Zn.

Brun et al. (2008) relaciona a disponibilidade de $\mathrm{S}$ com a qualidade e quantidade de matéria orgânica presente no solo, fato que explica os maiores valores desse nutriente com o aumento das doses de biossólido neste estudo.

\section{CONCLUSÕES}

- A aplicação de biossólido apresentou efeito positivo sobre o crescimento de S. terebinthifolius, L. glyptocarpa e $P$. dubium. Entretanto, na dose de 8 L/cova, a aplicação de biossólido prejudicou o crescimento de S. multijuga.

- E. contortisiliquum apresentou maior incremento em altura sob fertilização mineral comparado aos tratamentos controle e com biossólido.

- A aplicação de biossólido aumentou os teores foliares de S em I. laurina, de P e Ca em L. glyptocarpa e de Mg em S. multijuga. Entretanto, houve uma redução nos teores de P em G. americana.

\section{AGRADECIMENTOS}

Os autores agradecem à Coordenação de Aperfeiçoamento de Pessoal de Nível Superior (CAPES) pela concessão da bolsa de mestrado ao primeiro autor e à Associação Pró-Gestão das Águas da Bacia Hidrográfica do Rio Paraíba do Sul (AGEVAP) pelo apoio financeiro concedido para elaboração dessa pesquisa. Agradecemos ainda à Companhia Estadual de Águas e Esgotos (CEDAE) pelo fornecimento do biossólido utilizado nessa pesquisa.

\section{REFERÊNCIAS BIBLIOGRÁFICAS}

Abreu, A. H. M. (2014). Biossólido na produção de mudas florestais (Dissertação de mestrado). Universidade Federal Rural do Rio de Janeiro, Seropédica.

Andrade, J. C., \& Abreu, M. F. (Eds.). (2006). Análise química de resíduos sólidos para monitoramento e estudos agroambientais (pp. 121-158). Campinas: Instituto Agronômico.

Andreoli, C. V. (1999). Uso e manejo do lodo de esgoto na agricultura e sua influência em características ambientais no agrossistema (Tese de doutorado). Universidade Federal do Paraná, Curitiba.

Andreoli, C. V., Von Sperling, M., \& Fernandes, F. (2001). Lodos de esgoto: tratamento e disposição final. Belo Horizonte: Departamento de Engenharia Sanitária e Ambiental, Universidade Federal de Minas Gerais; Companhia de Saneamento do Paraná. 
Baggio, A. J. (1988). Aroeira como potencial para usos múltiplos na propriedade rural. Boletim de Pesquisa Florestal, 17, 25-32.

Barbosa, G. M. C., \& Tavares Filho, J. (2006). Uso agrícola do lodo de esgoto: influências nas propriedades químicas e física do solo, produtividade e recuperação de áreas degradadas. Semina: Ciências Agrárias, 27(4), 565-580. http://dx.doi.org/10.5433/1679-0359.2006v27n4p565.

Bataglia, O. C., Furlani, A. M. C., Teixeira, J. P. F., Furlani, P. R., \& Gallo, J. R. (1983). Métodos de análise química de plantas (Boletim Técnico, No. 78). Campinas: Instituto Agronômico.

Berton, R. S., \& Nogueira, T. A. R. (2010). Uso de lodo de esgoto na agricultura. In A. R. Coscione, T. A. R. Nogueira \& A. M. M. Pires (Eds.), Uso agrícola de lodo de esgoto: avaliação após a resolução $n^{\circ} 375$ do CONAMA (Cap. 2, pp. 31-50). Botucatu: FEPAF.

Bettiol, W., \& Camargo, O. (2006). Lodo de esgoto: impactos ambientais na agricultura. Jaguariúna: Embrapa Meio Ambiente.

Bettiol, W., \& Carvalho, P. C. T. (1982). Utilização de lodo de esgoto primário e fertilizante organomineral IPT na cultura do milho. Fertilizantes, 4(1), 14-15.

Brasil. Ministério das Cidades. Secretaria Nacional de Saneamento Ambiental. Sistema Nacional de Informações sobre Saneamento - SNIS. (2016). Diagnóstico dos serviços de água e esgotos: 2014. Brasília: SNSA/MCIDADES.

Brun, F. G. K., Szymczak, D. A., Brun, E. J., Londero, E. K., \& Schumacher, M. V. (2008). Aspectos nutricionais de exemplares de guabiju (Myrcianthes pungens (Berg) Legrand) implantados na arborização viária de Santa Maria - RS. Projeto de Pesquisa, 2(1), 7-15.

Caldeira, M. V. W., Peroni, L., Gomes, D. R., Delarmelina, W. M., \& Trazzi, P. A. (2012). Diferentes proporções de biossólido na composição de substratos para produção de mudas de timbó (Ateleia glazioveana Baill.). Scientia Forestalis, 40(93), 15-22.

Campos, F. S., \& Alves, M. C. (2008). Uso de lodo de esgoto na reestruturação de solo degradado. Revista Brasileira de Ciência do Solo, 32(4), 1389-1397. http://dx.doi.org/10.1590/S010006832008000400003.

Campos, T. O. (2017). Lixiviação de metais pesados e macronutrientes em solos com biossólido de lodo de esgoto (Dissertação de mestrado). Universidade Federal Rural do Rio de Janeiro, Seropédica.

Carpanezzi, A. A., Brito, J. O., \& Jark Filho, P. F. W. (1976). Teor de macro e micronutrientes em folhas de diferentes idades de algumas essências florestais nativas. Anais da Escola Superior de Agricultura. Luiz de Queiroz, 33, 225-232. http://dx.doi.org/10.1590/S0071-12761976000100018.

Colodro, G., \& Espíndola, C. R. (2006). Alterações na fertilidade de um latossolo degradado em resposta à aplicação de lodo de esgoto. Acta Scientiarum. Agronomy, 28(1), 1-5. http://dx.doi.org/10.4025/actasciagron.v28i1.1137.

Colodro, G., Espíndola, C. R., Cassiolato, A. M. R., \& Alves, M. C. (2007). Atividade microbiana em um latossolo degradado tratado com lodo de esgoto. Revista Brasileira de Engenharia Agrícola e Ambiental, 11(2), 195-198. http://dx.doi.org/10.1590/S1415-43662007000200010.

Dawkins, K., \& Esiobu, N. (2016). Emerging insights on Brazilian Pepper Tree (Schinus terebinthifolius) invasion: the potential role of soil microorganisms. Frontiers of Plant Science, 7, 712. PMid:27252726. http://dx.doi.org/10.3389/fpls.2016.00712.

Empresa Brasileira de Pesquisa Agropecuária - EMBRAPA. Centro Nacional de Pesquisa de Solos CNPS. (1997). Manual de métodos de análise de solo (2. ed. rev. atual., Documentos, No. 1). Rio de Janeiro: EMBRAPA-CNPS.

Godoy, L. C. (2013). A logística na destinação do lodo de esgoto. Revista Científica On-line Tecnologia, Gestão e Humanismo, 2(1), 79-90. Recuperado em 03 de julho de 2018, de http://www.fatecguaratingueta.edu.br/revista/index.php/RCO-TGH/article/view/43/27

Gonçalves, J. L. M. (1994). Relatório de pesquisas sobre nutrição mineral de espécies nativas. Piracicaba: Convênio CESP-ESALQ-IPEF. 25 p.

Gonçalves, J. L. M., Santarelli, E. G., Moraes Netto, S. P., Manara, M. P., \& Stape, J. L. (2000). Produção de mudas de espécies nativas: substrato, nutrição, sombreamento e fertilização. In J. L. M. Gonçalves \& V. Benedetti (Eds.), Nutrição e fertilização florestal (Cap. 11, pp. 309-350). Piracicaba: IPEF.

Guedes, M. C., \& Poggiani, F. (2003). Variação dos teores de nutrientes foliares em eucalipto fertilizado com biossólido. Scientia Forestalis, (63), 188-201.

Guedes, M. C., Andrade, C. A., Poggiani, F., \& Mattiazzo, M. E. (2006). Propriedades químicas do solo e nutrição do eucalipto em função da aplicação de lodo de esgoto. Revista Brasileira de Ciência do Solo, 30(2), 267-280. http://dx.doi.org/10.1590/S0100-06832006000200008. 
Instituto Nacional de Meteorologia - INMET. (2017). Dados climáticos da Estação Automática Ecologia Agrícola, Seropédica, Km 47-RJ. Recuperado em 18 de janeiro de 2017, de http://www.inmet.gov.br/portal/index.php?r=estacoes/estacoesautomaticas

Lima Filho, P. (2015). Biossólido na restauração florestal: formação de mudas e adubação de plantio (Dissertação de mestrado). Universidade Federal Rural do Rio de Janeiro, Seropédica.

Macho, S. A., \& Figueiredo Filho, A. (2006). Dendrometria (2. ed., 316 p.). Guarapuava: UNICENTRO.

Maio, M. M., Sampaio, R. A., Nascimento, A. L., Prates, F. B. S., Rodrigues, M. N., Silva, H. P., Dias, A. N., \& Freitas, C. E. S. (2011). Atributos físicos do solo, adubado com lodo de esgoto e silicato de cálcio e magnésio. Revista Ceres, 58(6), 823-830. http://dx.doi.org/10.1590/S0034-737X2011000600021.

Maria, I. C., Chiba, M. K., Costa, A., \& Berton, R. S. (2010). Sewage sludge application to agricutural land as soil physical conditioner. Revista Brasileira de Ciência do Solo, 34(3), 967-974. http://dx.doi.org/10.1590/S0100-06832010000300038.

Munns, R. (2002). Comparative physiology of salt and water stress. Plant, Cell \& Environment, 25(2), 239250. PMid:11841667. http://dx.doi.org/10.1046/j.0016-8025.2001.00808.x.

Paiva, A. V. (2005). Efeitos da aplicação de biossólido no crescimento e nutrição mineral de mudas de quatro espécies florestais nativas utilizadas na arborização urbana (Tese de doutorado). Escola Superior de Agricultura "Luiz de Queiroz", Universidade de São Paulo, Piracicaba.

Rocha, G. N., Gonçalves, J. L. M., \& Moura, I. M. (2004). Mudanças da fertilidade do e crescimento de um povoamento de Eucalyptus grandis fertilizado com biossólido. Revista Brasileira de Ciência do Solo, 28(4), 623-639. http://dx.doi.org/10.1590/S0100-06832004000400005.

Santos, F. M. (2015). Produção de biomassa e eficiência de uso dos nutrientes em plantios puros e mistos de Eucalyptus urograndis e Acácia mangium Willd (Dissertação de mestrado). Universidade Federal Rural do Rio de Janeiro, Seropédica.

Silva, B. V. N., \& Pinto, L. V. A. (2010). Potencial do uso do lodo de esgoto como adubo orgânico em cobertura de espécies florestais nativas plantadas em área degradadas por pastagem. Revista Agrogeoambiental, 2(1), 50-56. http://dx.doi.org/10.18406/2316-1817v2n12010251.

Silva, F. C., Boaretto, A. E., Berton, R. S., Zotelli, H. B., Pexe, C. A., \& Bernardes, E. M. (2001). Efeito de lodo de esgoto na fertilidade de um Argissolo Vermelho-Amarelo cultivado com cana-de-açúcar. Pesquisa Agropecuária Brasileira, 36(5), 831-840. http://dx.doi.org/10.1590/S0100$204 \times 2001000500014$.

Silva, I. R., Furtini Neto, A. E., Curi, N., \& Vale, F. R. (1997). Crescimento inicial de quatorze espécies florestais nativas em resposta à adubação potássica. Pesquisa Agropecuária Brasileira, 32(2), 205212.

Silva, P. H. M., Poggiani, F., Gonçalves, J. L. M., Stape, J. L., \& Moreira, R. M. (2008a). Crescimento de Eucalyptus grandis tratado com diferentes doses de lodos de esgoto úmido e seco, condicionados com polímeros. Scientia Forestalis, 36(77), 79-88.

Silva, P. H. M., Poggiani, F., Gonçalves, J. L. M., \& Stape, J. L. (2008b). Volume de madeira e concentração foliar de nutrientes em parcelas experimentais de Eucalyptus grandis fertilizadas com lodos de esgoto úmido e seco. Revista Árvore, 32(5), 845-854. http://dx.doi.org/10.1590/S010067622008000500009.

Simonete, M. A., Kiehl, J. C., Andrade, C. A., \& Teixeira, C. F. A. (2003). Efeito do lodo de esgoto em um Argissolo e no crescimento e nutrição de milho. Pesquisa Agropecuária Brasileira, 38(10), 1187-1195. http://dx.doi.org/10.1590/S0100-204X2003001000008.

Tester, M., \& Davenport, R. (2003). Na+ tolerance and $\mathrm{Na}^{+}$transport in higher plants. Annals of Botany, 91(5), 503-527. PMid:12646496. http://dx.doi.org/10.1093/aob/mcg058.

Contribuição dos autores: MVS: conceituação, curadoria de dados, análise formal, obtenção de financiamento, investigação, metodologia, visualização, escrita - primeira redação; GMC: conceituação, análise formal, obtenção de financiamento, investigação, metodologia, administração do projeto, recursos, supervisão, escrita - revisão e edição; PSSL: conceituação, investigação, metodologia, recursos, escrita - revisão e edição; ASR: conceituação, investigação, escrita - revisão e edição; EVS: conceituação, obtenção de financiamento, escrita - revisão e edição; TOCB: curadoria de dados, análise formal, investigação, visualização, escrita - revisão e edição. 